\title{
Position of phonetic components may influence how written words are processed in the brain: Evidence from Chinese phonetic compound pronunciation
}

\author{
JANET H. HSiAO ANd TianYin LiU \\ University of Hong Kong, Hong Kong
}

\begin{abstract}
Previous studies have shown a right-visual-field (RVF)/left-hemisphere (LH) advantage in Chinese phonetic compound pronunciation. Here, we contrast the processing of two phonetic compound types: a dominant structure in which a semantic component appears on the left and a phonetic component on the right (SP characters), and a minority structure with the opposite arrangement (PS characters). We show that this RVF/LH advantage was observed only in SP character pronunciation, but not in PS character pronunciation. This result suggests that SP character processing is more LH lateralized than is PS character processing and is consistent with corresponding ERP N170 data. This effect may be due to the dominance of SP characters in the lexicon, which makes readers opt to obtain phonological information from the right of the characters. This study thus shows that the overall information distribution of word components in the lexicon may influence how written words are processed in the brain. Supplemental materials for this article may be downloaded from http://cabn .psychonomic-journals.org/content/supplemental.
\end{abstract}

It has long been observed that the processes of visual word recognition in alphabetic languages such as English are lateralized to the left hemisphere (LH). Data from fMRI studies show a region inside the left fusiform area (visual word form area) responding selectively to written words (e.g., McCandliss, Cohen, \& Dehaene, 2003; although some have argued that this region also responds to stimuli other than words and in tasks other than reading; see, e.g., Price \& Devlin, 2003). ERP studies also have shown that words elicit a larger N170 in the LH, as compared with strings of symbols (e.g., Maurer, Brandeis, \& McCandliss, 2005). Consistent with these findings, a classical right-visual-field (RVF)/LH advantage in reading English words has been consistently reported, demonstrated first in tachistoscopic recognition (e.g., Bryden \& Rainey, 1963) and subsequently in other word recognition tasks, including lexical decision (Faust, Babkoff, \& Kravetz, 1995) and word-naming (Brysbaert \& d'Ydewalle, 1990) tasks. This RVF/LH advantage has been argued to be linked to the LH superiority in language processing - in particular, phonological processing - and has been shown to interact with sex and handedness (e.g., Voyer, 1996).

In contrast, the recognition of Chinese characters, a logographic writing system, has been shown to have a leftvisual-field (LVF)/right-hemisphere (RH) advantage in tasks that mainly depend on the processing of orthography/ word forms (e.g., Cheng \& Yang, 1989; Tzeng, Hung, Cotton, \& Wang, 1979) and an RVF/LH advantage in tasks that mainly depend on the processing of phonology (e.g., Weekes \& Zhang, 1999). ${ }^{1}$ For example, Tzeng et al. showed an LVF/RH advantage in tachistoscopic recognition of single Chinese characters; this effect has been argued to reflect the RH superiority in handling holistic pattern recognition (e.g., Tzeng et al., 1979) or a more effective activation of lexical knowledge of Chinese characters in the RH, as compared with the $\mathrm{LH}$, given that no laterality effect was found for pseudo- and noncharacters (Cheng \& Yang, 1989). As for phonological processing, Weekes and Zhang (1999) reported phonological priming effects on phonetic compound recognition when the characters were presented in the RVF, but not when presented in the LVF. Yang and Cheng (1999) also showed that in tachistoscopic recognition of Chinese characters, when the orthographic similarity of two alternative items for choice was manipulated, there was an LVF advantage; in contrast, when the phonological similarity of two alternative items for choice was manipulated, there was an RVF advantage. Consistent with the behavioral data, fMRI and ERP studies of Chinese character recognition have generally exhibited a more bilateral or RH-lateralized activation in the visual system, as compared with English word recognition (e.g., Liu \& Perfetti, 2003; Tan et al., 2001; Tan et al., 2000). Hence, Chinese character recognition seems 
to involve an interaction between the processing preferences of the RH (orthography) and the LH (phonology).

The difference in hemispheric lateralization between English word and Chinese character recognition has been argued to be due to the RH superiority in handling the holistic pattern recognition that is required in Chinese character recognition (e.g., Tzeng et al., 1979). Nevertheless, a recent study showed a decreased holistic-processing effect in Chinese character recognition in Chinese readers, as compared with non-Chinese readers (Hsiao \& Cottrell, 2009), which is inconsistent with this claim. Alternatively, Maurer and McCandliss (2007) proposed the phonological-mapping hypothesis and argued that, given that phonological processes are typically LH lateralized (e.g., Rumsey et al., 1997), specialized processing of visual words in visual brain areas also becomes LH lateralized. Accordingly, they pointed out that the LH lateralization in English word recognition may be related to the influence of grapheme-phoneme conversion established when one learns to read, and this modulation should be less pronounced in logographic scripts such as Chinese.

However, some Chinese characters do have a phonological component that has a functionality similar to the grapheme-phoneme mapping in English words. ${ }^{2}$ These characters are a dominant type of Chinese characters, phonetic compounds, which make up about $81 \%$ of the 7,000 most frequent characters (Li \& Kang, 1993). A phonetic compound consists of a semantic radical that usually reflects character meaning and a phonetic radical that typically provides partial information about character pronunciation (see Figure 1). Thus, a phonetic compound character may also have LH phonological modulation when one learns to read, due to the requirement of mapping its phonetic component to the corresponding pronunciation as part of the character pronunciation, and consequently its processing may become LH lateralized. Consistent with this claim, Weekes, Chen, and Lin (1998) compared phonological-priming effects in the recognition of phonetic compound characters and integrated characters (i.e., characters that do not contain separate radical components and, thus, do not have a phonetic radical) and showed that there was a significant phonological-priming effect in phonetic compound character recognition, but not in the recognition of integrated characters (see also Weekes \& Zhang, 1999).

Among these phonetic compound characters, different structures exist, including left-right, top-bottom, concentric, and other irregular structures (Figure 1). Here, we

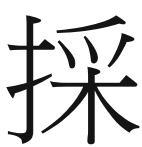

S P

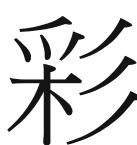

P S

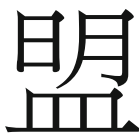

Top-bottom

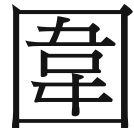

Concentric
Figure 1. Examples of Chinese phonetic compound characters: left-right (semantic-phonetic [SP] and PS characters), topbottom, and concentric. The SP and PS characters have the same phonetic radical and the same pronunciation [coi3] in Pinyin. hypothesize that phonetic compounds in different structures may be processed differently in the brain, because the phonologically important part of the characters - that is, the phonetic radical - appears in different locations in different character structures, and Chinese readers may opt to use phonological information from a location where useful information is most likely to be obtained. For example, about two thirds of the phonetic compounds have a left-right structure (Hsiao \& Shillcock, 2006); among these left-right structured phonetic compounds, about $90 \%$ of them have the semantic radical on the left and the phonetic radical on the right (SP characters), and the other $10 \%$ have the opposite arrangement (PS characters; Hsiao \& Shillcock, 2006; see Figure 1). Since SP characters are a dominant structure among phonetic compounds, Chinese readers may opt to use phonological information from the right side of a character more often than from any other locations. Thus, the automaticity of phonological processing in SP character recognition may be superior to that in PS character recognition. According to the phonologicalmapping hypothesis (Maurer \& McCandliss, 2007), consequently, the processing of SP characters may involve more LH phonological modulation, as compared with phonetic compounds in other structures. This examination is not possible in any alphabetic languages, since in words of alphabetic languages, phonological information is distributed across the whole word, rather than located on one side. The separation of semantic and phonetic components and the dominance of the SP structure in the lexicon provide this unique opportunity for examining whether the position of the phonetic components influences how written words are processed in the brain.

Thus, here our aim is to investigate whether the recognition of Chinese characters in different structures involves different hemispheric lateralization by examining the processing of two types of phonetic compounds with contrasting structures: SP and PS characters. We conducted a divided visual field study of SP and PS character naming, with pairs of SP and PS characters well matched in terms of character frequency, phonetic radical, visual complexity (defined by the number of strokes), and pronunciation. If the automaticity of phonological processing in SP character recognition is superior to that in PS character recognition, due to the dominance of the SP structure, the processing of SP characters will involve more LH phonological modulation, as compared with PS characters, and consequently there will be a stronger RVF/LH advantage in naming SP characters, as compared with PS characters. In contrast, if character processing is not influenced by the overall information distribution of word components in the lexicon, there will be a comparable RVF/LH advantage in naming SP and PS characters.

\section{METHOD}

\section{Materials}

The materials consisted of 76 pairs of Chinese phonetic compound characters that have a left-right configuration in traditional Chinese fonts. Characters were carefully chosen so that each SP-PS character pair shared the same phonetic radical and also had 


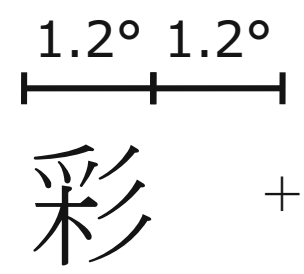

Figure 2. Divided visual field presentation. During the experiment, characters were presented $1.2^{\circ}$ of visual angle away from the center of the screen to avoid the foveal vision.

the same pronunciation in Cantonese (see Hsiao \& Shillcock, 2005; see Figure 1). Characters in the materials were within a medium- to high-frequency range of usage in Hong Kong society during the 1980 s and 1990s, according to a Chinese character frequency database (Ho, 1998); the database stored 663,463 characters used in Hong Kong during the 1980s and 1990s, and the median frequency among all characters was 16 , whereas the median for the characters used in this study was 26 . Also, there was no significant difference between SP and PS character pairs in terms of character frequency and number of strokes (paired $t$ test, n.s.; see Supplemental Table 1 for a list of stimuli).

\section{Participants}

We recruited 40 native Traditional Chinese readers from Hong Kong (Cantonese speaking), among whom 15 were male and 25 were female. They were all students or staff members at the University of Hong Kong, with a mean age of 21 years 11 months; all were right-handed according to the Edinburgh handedness inventory (Oldfield, 1971), and all had normal or corrected-to-normal vision (i.e., with glasses or contact lenses). The participants received some honorarium for their participation.

\section{Design}

The design consisted of two within-subjects variables: position of the phonetic radical (PS vs. SP) and visual field (LVF vs. RVF). The dependent variable was the time taken to start a correct pronunciation (correct response times). In order to avoid any priming effect, each participant saw each character only once during the experiment, in either the LVF or the RVF; the visual field presentation condition for each character was counterbalanced among participants. In other words, in the experiment, two Latin-square groups were created, with characters presented in the opposite visual field be- tween the two groups. To account for this difference in Latin-square group among the participants, we included a Latin-square variable as a between-subjects variable in the design (Brysbaert, 2007; Pollatsek \& Well, 1995). The participants were asked to sit in front of a computer screen, at a viewing distance of $50 \mathrm{~cm}$; under this viewing distance, each character subtended about $1.2^{\circ}$ of visual angle. To avoid presenting characters within the foveal vision, characters were presented $1.2^{\circ}$ of visual angle away from the center of the screen (see Figure 2).

\section{Procedure}

Figure 3 shows the experimental procedure. Each naming trial started with a 500-msec central fixation cross; the participants were told to look at the cross when it appeared. The fixation cross was followed by a 150 -msec presentation of the target character in either the LVF or the RVF (Figure 2). The participants were asked to pronounce the character as quickly and accurately as possible; their response time and accuracy were recorded. A central fixation cross was presented after the presentation of the character, and the fixation cross disappeared after the onset of the participants' pronunciation. The screen then turned blank until the experimenter pressed a button to start the next trial. Occasionally, on some trials, a very small digit was presented instead of a character for $90 \mathrm{msec}$ at the center of the screen; the participants were also asked to name the digit when it appeared. We included this design to make sure that they were accurately looking at the fixation cross before the presentation of the characters/digits. We removed data from the participants who had an accuracy lower than .8 in the digit-naming task, since this might suggest that the participants had not always accurately fixated at the center of the screen during the experiment (e.g., Brysbaert, 1994; Hunter, Brysbaert, \& Knecht, 2007). ${ }^{3}$

The recorded response time was the time difference between the onset of the character presentation and the onset of the participants' pronunciation; it was measured through a voice key trigger in the PST serial response box, and the experiment was controlled through the E-Prime software (Psychology Software Tools, Inc.). Only the response times on the trials with a correct pronunciation were analyzed. In the experiment, the presentation order of each pair of PS and SP characters and the presented visual field for each character were counterbalanced among the participants. Before the experiment, the participants performed a practice session with characters not included in the materials.

\section{RESULTS}

In the accuracy data, the participants had high naming accuracy, since the stimuli were medium- to high-frequency characters. The overall accuracy was .946 , and it was .940 and .952 for naming PS and SP characters, respectively. There was no significant difference in accuracy between naming PS and SP characters (paired $t$ test, n.s.).

In the response time data, the results showed a significant main effect of position of the phonetic radical $[F(1,38)=$

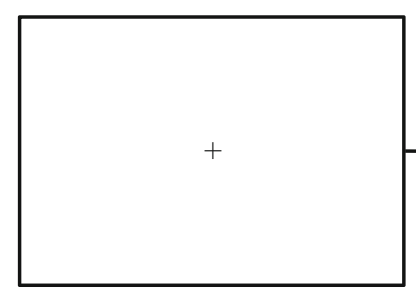

$500 \mathrm{msec}$

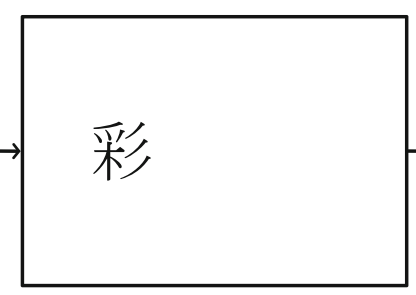

$150 \mathrm{msec}$

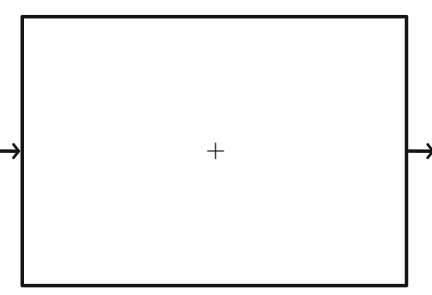

Until pronunciation onset

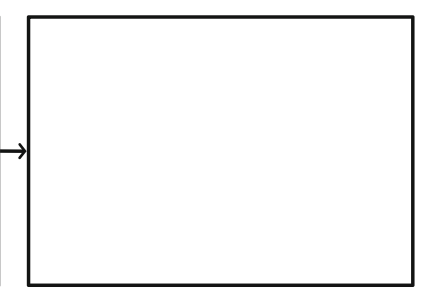

Figure 3. Experimental procedure. 
27.133, $p<.001$ ], since SP characters were named more quickly than PS characters, and a significant main effect of visual field $[F(1,38)=8.345, p<.01]$, since characters were named more quickly when they were presented in the RVF, as compared with when they were presented in the LVF. In addition, there was a significant interaction between visual field and position of the phonetic radical $[F(1,38)=5.909, p<.05]$ : There was an RVF advantage in naming SP characters $[F(1,38)=13.868, p=.001$; see Figure 4]. In contrast, no visual field difference was observed in naming PS characters $(F<1)$. When we examined the data in the two visual fields separately, the participants were significantly faster in naming SP characters than in naming PS characters when the characters were presented in the RVF $[F(1,38)=27.213, p<.001$; see Figure 4]; in contrast, this difference was not significant when the characters were presented in the LVF. The results thus showed that the processing of SP and PS characters involves different hemispheric lateralization. In other words, the position of the phonetic component influences how Chinese phonetic compounds are processed in the brain. This result suggests that the overall information distribution of word components in the lexicon may influence how visual words are processed in the brain.

\section{DISCUSSION}

Here, our aim was to investigate whether the position of phonetic components influences how visual words are processed in the brain through examining the processing of two types of phonetic compound characters with contrasting structures: SP characters (i.e., a semantic component on the left and a phonetic component on the right) and PS characters (i.e., the opposite arrangement). We hypoth-

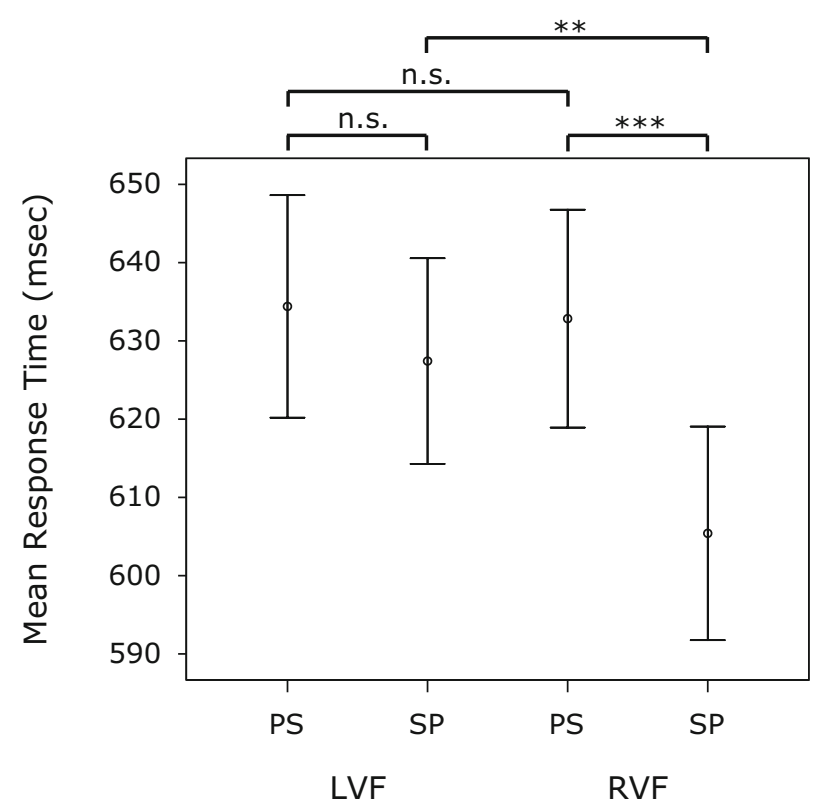

Figure 4. Results of the naming experiment. The error bars show standard errors $\left({ }^{* *} p<.01 ;{ }^{* * * *} p<.001\right)$. P, phonetic; S, semantic; LVF, left visual field; RVF, right visual field. esized that the dominance of the SP structure in the lexicon makes Chinese readers opt to use the right component of a character as the location from which useful phonological information is most likely to be obtained and, thus, that phonological information can be more efficiently extracted and processed in SP characters, as compared with PS characters. Consequently, the processing of SP characters involves more LH phonological modulation (Maurer \& McCandliss, 2007) and, thus, is more LH lateralized, as compared with PS characters. Our result was consistent with this hypothesis. It showed a significant interaction between character type and position of the phonetic component: There was an RVF/LH advantage in naming SP characters; in contrast, no visual field difference was observed in naming PS characters (Figure 4). When data in the LVF and the RVF were examined separately, there was no significant difference in response times between naming SP and PS characters when they were presented in the LVF/RH; in contrast, when they were presented in the RVF/LH, SP characters were pronounced significantly faster than PS characters (Figure 4). This result supports the hypothesis that the observed difference in hemispheric lateralization in naming SP and PS characters was due to the LH phonological modulation in SP character processing, but not in PS character processing.

The present results are consistent with an ERP study of Chinese character recognition contrasting the processing of SP and PS characters reported by Hsiao, Shillcock, and Lee (2007). In the ERP study, participants performed a sequential homophone judgment task, with characters being centrally presented one at a time while their EEGs were recorded; the characters spanned less than $1^{\circ}$ of visual angle and, thus, fell within the foveal vision. As in the present study, the materials consisted of 75 pairs of SP and PS characters, and each pair shared the same phonetic radical and had the same pronunciation, and the members of the pairs were matched in terms of token frequency and semantic radical visual complexity (defined by number of strokes). The results showed a significant interaction between character type (SP vs. PS) and hemisphere in N170 amplitude: SP character elicited a larger N170 (N1) amplitude than did PS characters in the LH, whereas PS characters elicited a larger N170 amplitude than did SP characters in the RH. Nevertheless, it was unclear whether N170 was actually larger in the LH than in the RH for SP characters, as compared with PS characters, since this analysis was not conducted in the study. Thus, here we reanalyzed the ERP data for SP and PS characters separately, in order to examine whether the processing of SP characters is more lateralized to the $\mathrm{LH}$, as compared with the processing of PS characters, as suggested by the present behavioral results.

In this ERP data reanalysis, instead of using an averaged mastoid (ear) reference (Hsiao et al., 2007), we used the common average (i.e., average over all scalp sites) as the reference, to avoid the concern that the mastoids are very close to the active region of the N170 component (i.e., the inferior-temporal region). In contrast, the common average reference has been recommended by recent guidelines for scalp electrophysiological research (Picton 
et al., 2000), on the basis of the theory that a constant zero average is maintained across the scalp (Bertrand, Perrin, \& Pernier, 1985). ${ }^{4}$ It also has been shown to be the reference that most optimally captures categorical and hemispheric differences in N170 (Joyce \& Rossion, 2005). We used the same artifact rejection methods as those reported in Hsiao et al. The N170 component was identified at around 180-220 msec poststimulus. Electrodes that had the most prominent peaks in N170 were selected for analysis and were paired between the two hemispheres. The electrodes selected for analyses in the $\mathrm{LH}$ were $\mathrm{PO} 7$ and PO5; those in the RH were PO8 and PO6. ${ }^{5}$ The N170 amplitude was measured over a 50 -msec window centered on the peak latency of the maximum amplitude in the grandaveraged data, separately for different conditions (Hsiao et al., 2007). The data included 16 male and 16 female participants.

Our reanalysis confirmed that there was a significant interaction between character type and hemisphere in N170 amplitude $[F(1,26)=16.265, p<.001]$; this effect did not interact with the electrodes we selected $[F(1,26)=1.505$, n.s.]. ${ }^{6}$ In addition, when we analyzed the data of SP and PS characters separately, we observed that SP characters elicited significantly larger N170 in the LH than in the $\mathrm{RH}[F(1,29)=4.982, p<.05$; this effect did not interact with the electrodes we selected, $F(1,29)<1$, n.s.]; in contrast, PS characters elicited N170 in both hemispheres with comparable amplitude $[F(1,27)=0.590$, n.s.; see Figure 5]. ${ }^{7}$ N170 amplitude has been shown to be a reliable measure for hemispheric asymmetry in the recognition of visual categories such as faces and words (Maurer et al., 2005; Mercure, Dick, Halit, Kaufman, \& Johnson, 2008), and correspondence between ERP and behavioral data can usually be observed even when different paradigms are used between them. For example, behaviorally, the RVF/LH advantage in English word processing in divided visual field studies (e.g., Brysbaert \& d'Ydewalle, 1990 ) is consistent with the larger N170 amplitude in the LH than in the RH when English words are centrally presented (e.g., Rossion, Joyce, Cottrell, \& Tarr, 2003); also, the LVF/RH advantage in face processing in the behavioral data (e.g., Levine \& Koch-Weser, 1982) is consistent
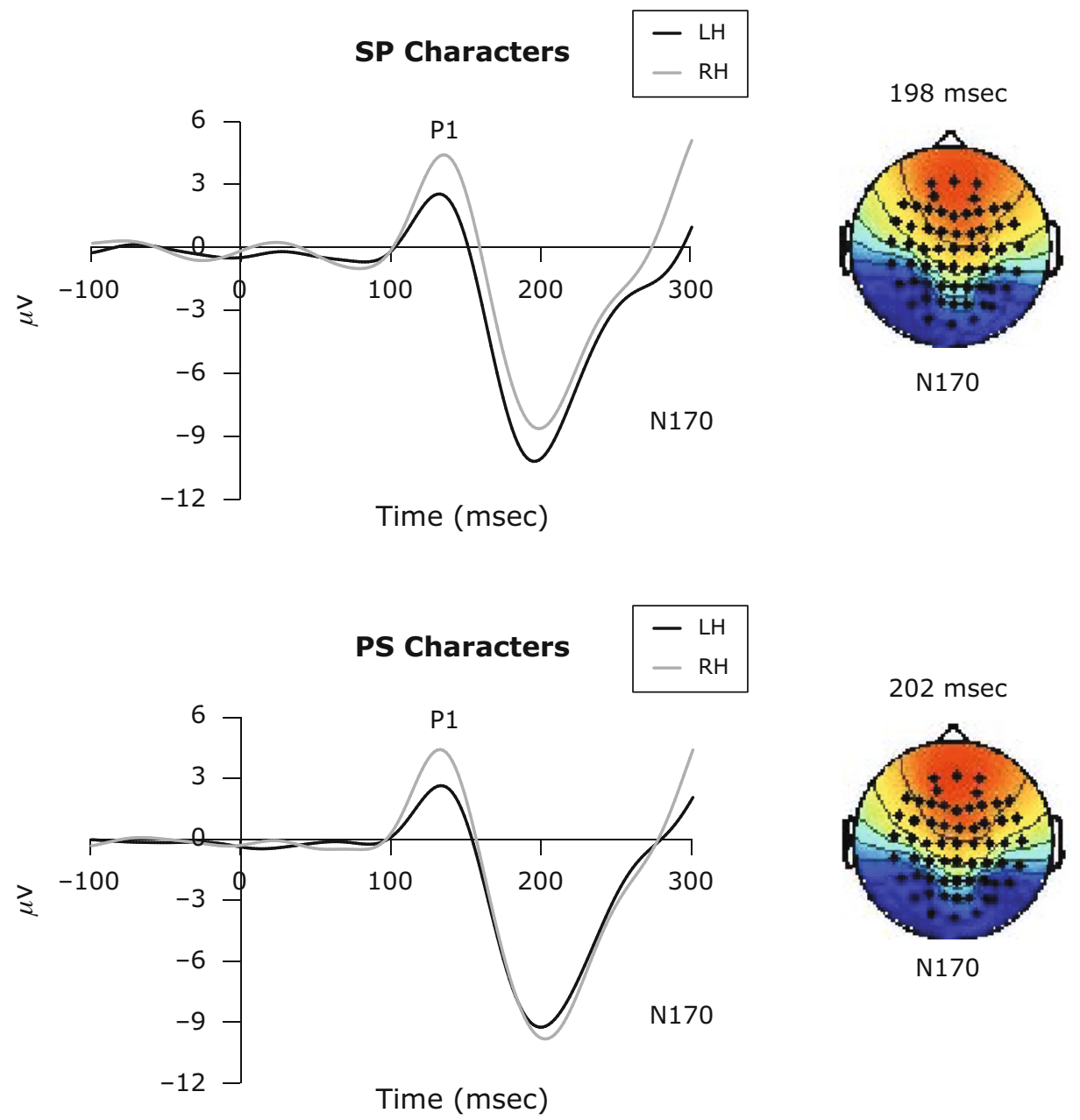

Figure 5. Corresponding ERP data (with characters centrally presented): semantic-phonetic (SP) characters elicited larger N170 amplitude in the left hemisphere (LH) than in the right hemisphere (RH); in contrast, PS characters elicited comparable N170 amplitude in both hemispheres. 
with the larger N170 amplitude in the RH than in the LH when faces are centrally presented (Rossion et al., 2003). Similarly, the ERP effect reported here matched well with the behavioral naming data; they provided different but complementary pieces of evidence showing that the processing of SP characters is more lateralized to the $\mathrm{LH}$, as compared with the processing of PS characters.

Here, we argue that the most parsimonious account for the observed processing differences between SP and PS characters in hemispheric asymmetry may be the dominance of SP characters in the lexicon, which makes Chinese readers opt to obtain phonological information from the right side of a character, and thus the automaticity of phonological processing in SP character recognition is superior to that in PS character recognition; consequently, SP character processing involves more LH phonological modulation (Maurer \& McCandliss, 2007), as compared with the processing of PS characters. ${ }^{8}$ The separation of semantic and phonetic components and the dominance of SP characters in the Chinese lexicon provide this unique opportunity to show how visual word processing can be influenced by the overall information distribution of word components in the lexicon. Here, we compared the processing of SP and PS characters because of their contrasting structures and the dramatic difference in type frequency in the lexicon (i.e., nine to one); we predict that when compared with other types of phonetic compounds that do not have a typical left-right structure, the processing of SP characters will still have a stronger LH lateralization, due to the dominance of SP characters in the lexicon, although this speculation requires further examination.

The difference in lateralization between the processing of SP and PS characters may also be explained by their difference in the regularity of the relationship between character pronunciation and pronunciation of the phonetic radical. It has been reported that among SP characters, there is a higher percentage of characters that have the same pronunciation as their phonetic radical (i.e., regular characters), as compared with PS characters (Hsiao \& Shillcock, 2006; see note 2). The phonetic radical of a regular character provides more useful information about the character pronunciation, as compared with that of an irregular character, whose pronunciation shares only the same onset or rhyme as or is completely different from the character pronunciation. Thus, the recognition of regular characters may involve more phonological processing, as compared with the recognition of irregular characters. This factor may also contribute to the lateralization difference between SP and PS characters, in addition to the dominance of SP characters in the lexicon.

Alternatively, our present results may also be explained under the split fovea assumption. Recent research has suggested that human foveal representation may be split along the vertical midline, with the two halves initially contralaterally projected to the two hemispheres (e.g., Brysbaert, 2004; Lavidor \& Walsh, 2004; for a review, see Ellis \& Brysbaert, 2010), as opposed to bilaterally projected, as was previously thought (e.g., Huber, 1962; Stone, Leicester, \& Sherman, 1973). Under this split fovea assumption, a centrally fixated SP character will have its phonetic radical initially projected to the $\mathrm{LH}$, where phonological processes are typically lateralized, whereas the phonetic radical of a PS character will be initially projected to the RH (see Figure 6). It has been shown that when people are viewing individual characters, they fixate at the center of the characters most often (e.g., Hsiao \& Cottrell, 2009). Thus, it is possible that when Chinese readers are learning to read individual characters, they receive more phonological modulation in the LH when learning to read SP characters, as compared with PS characters, and this learning experience may result in different hemispheric lateralization in processing SP and PS characters. Note, however, that Jordan and Paterson (2009) have recently questioned the viability of the split fovea theory by pointing out potential problems in maintaining accurate fixations in experiments that required participants to fixate a particular location within a centrally presented word, and in the accuracy of reporting results from previous studies that were claimed to support the split fovea theory (see also Jordan, Paterson, Kurtev, \& Xu, 2009, and the reply in Ellis \& Brysbaert, 2010). Thus, it remains controversial whether foveal representation is split and initially contralaterally projected to different hemispheres; whether the effect observed here is due to the dominance of SP characters in the lexicon or the direct access of the phonetic component of an SP character to the LH according to the split fovea claim also requires further examination.

In summary, here we show that the position of phonetic components influences how written words are processed in the brain. We provide both behavioral and ERP data and show that the two types of Chinese phonetic compound characters with opposite phonetic component positions, SP and PS characters, are processed differently in the brain: The processing of SP characters is lateralized to the $\mathrm{LH}$, whereas the processing of PS characters is bilaterally distributed. We argue that this result may be due to the dominance of SP characters in the lexicon, which makes Chinese readers opt to obtain phonological information
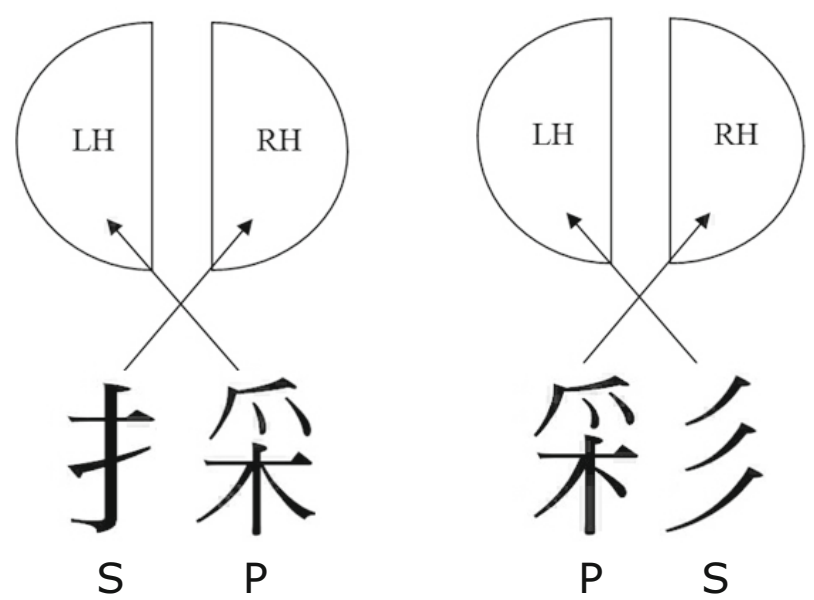

Figure 6. Contralateral projection of the two radicals when one learns to read semantic-phonetic (SP) and PS characters, according to the split fovea claim. $\mathrm{LH}$, left hemisphere; $\mathrm{RH}$, right hemisphere. 
from the right side of a character, and that, consequently, the processing of SP characters receives more LH phonological modulation, as compared with the processing of PS characters, and results in more LH lateralization. The separation of semantic and phonetic components in Chinese orthography and the existence of a dominant structure (SP characters) provide a unique opportunity for this examination; it shows that the overall information distribution of word components in the lexicon can influence how written words are processed in the brain.

\section{AUTHOR NOTE}

We are grateful to the HKU Seed Funding Program for Basic Research (Project 10400471 to J.H.H.) and the Research Grant Council of Hong Kong (Project HKU 744509H to J.H.H.). We thank the editor and three anonymous reviewers for helpful comments. Correspondence should be addressed to J. H. Hsiao, Department of Psychology, University of Hong Kong, 604 Knowles Building, Pokfulam Road, Hong Kong (e-mail: jhsiao@hku.hk orkanalty@gmail.com).

\section{REFERENCES}

Bertrand, O., Perrin, F., \& Pernier, J. (1985). A theoretical justification of the average reference in topographic evoked potential studies. Electroencephalography \& Clinical Neurophysiology, 62, 462-464.

Bryden, M. P., \& Rainey, C. A. (1963). Left-right differences in tachistoscopic recognition. Journal of Experimental Psychology, 66, 568-571.

BRYSBAERT, M. (1994). Interhemispheric transfer and the processing of foveally presented stimuli. Behavioural Brain Research, 64, 151161.

BRYSBAERT, M. (2004). The importance of interhemispheric transfer for foveal vision: A factor that has been overlooked in theories of visual word recognition and object perception. Brain \& Language, 88, 259267.

BrysbaerT, M. (2007). "The language-as-fixed-effect fallacy": Some simple SPSS solutions to a complex problem (Ver. 2.0) [Tech. Rep.]. London: Royal Holloway, University of London.

Brysbaert, M., \& D'Ydewalle, G. (1990). Tachistoscopic presentation of verbal stimuli for assessing cerebral dominance: Reliability data and some practical recommendations. Neuropsychologia, 28, 443-455.

Cheng, C. M., \& YAng, M. J. (1989). Lateralization in the visual perception of Chinese characters and words. Brain \& Language, 36, 669689.

DiEN, J. (1998). Issues in the application of the average reference: Review, critiques, and recommendations. Behavior Research Methods, Instruments, \& Computers, 30, 34-43.

Ellis, A. W., \& Brysbaert, M. (2010). Split fovea theory and the role of the two cerebral hemispheres in reading: A review of the evidence. Neuropsychologia, 48, 353-365.

Faust, M., Babkoff, H., \& Kravetz, S. (1995). Linguistic processes in the two cerebral hemispheres: Implications for modularity vs interactionism. Journal of Clinical \& Experimental Neuropsychology, 17, 171-192.

Ho, H. H. (1998). Hong Kong, Mainland China and Taiwan: Chinese character frequency-A trans-regional, diachronic survey. Retrieved July 4, 2009, from http://arts.cuhk.edu.hk/Lexis/chifreq/.

Hsiao, J. H., \& Cottrell, G. W. (2009). Not all visual expertise is holistic, but it may be leftist: The case of Chinese character recognition. Psychological Science, 20, 455-463.

HsiaO, J. H., \& SHILlCocK, R. (2005). Foveal splitting causes differential processing of Chinese orthography in the male and female brain. Cognitive Brain Research, 25, 531-536.

Hsiao, J. H., \& Shillcock, R. (2006). Analysis of a Chinese phonetic compound database: Implications for orthographic processing. Journal of Psycholinguistic Research, 35, 405-426.

Hsiao, J. H., Shillcock, R., \& Lee, C. (2007). Neural correlates of foveal splitting in reading: Evidence from an ERP study of Chinese character recognition. Neuropsychologia, 45, 1280-1292.
Huber, A. (1962). Homonymous hemianopia after occipital lobectomy. American Journal of Ophthalmology, 54, 623-629.

Hunter, Z. R., Brysbaert, M., \& KNecht, S. (2007). Foveal word reading requires interhemispheric communication. Journal of Cognitive Neuroscience, 19, 1373-1387.

JORDAN, T. R., \& PATERSON, K. B. (2009). Re-evaluating split-fovea processing in word recognition: A critical assessment of recent research. Neuropsychologia, 47, 2341-2353.

Jordan, T. R., Paterson, K. B., Kurtev, S., \& Xu, M. (2009). Do fixation cues ensure fixation accuracy in split-fovea studies of word recognition? Neuropsychologia, 47, 2004-2007.

Joyce, C., \& Rossion, B. (2005). The face-sensitive N170 and VPP components manifest the same brain processes: The effect of reference electrode site. Clinical Neurophysiology, 116, 2613-2631.

KATZNELSON, R. D. (1981). EEG recording, electrode placement, and aspects of generator localization. In P. L. Nunez (Ed.), Electric fields of the brain: The neurophysics of EEG (pp. 76-213). New York: Oxford University Press.

LAVIDOR, M., \& WALSH, V. (2004). The nature of foveal representation. Nature Reviews Neuroscience, 5, 729-735.

Levine, S. C., \& КосH-Weser, M. P. (1982). Right hemisphere superiority in the recognition of famous faces. Brain \& Cognition, 1, 1022.

LI, Y., \& KANG, J. S. (1993). Analysis of phonetics of the ideophonetic characters in modern Chinese [In Chinese]. In Y. Chen (Ed.), Information analysis of usage of characters in modern Chinese (pp. 84-98). Shanghai: Shanghai Education.

Liu, Y., \& Perfetti, C. A. (2003). The time course of brain activity in reading English and Chinese: An ERP study of Chinese bilinguals. Human Brain Mapping, 18, 167-175.

Maurer, U., Brandeis, D., \& McCandliss, B. D. (2005). Fast, visual specialization for reading in English revealed by the topography of the N170 ERP response. Behavioral \& Brain Functions, 1, 13.

Maurer, U., \& McCandliss, B. D. (2007). The development of visual expertise for words: The contribution of electrophysiology. In E. L. Grigorenko \& A. J. Naples (Eds.), Single-word reading: Cognitive, behavioral and biological perspectives (pp. 43-63). Mahwah, NJ: Erlbaum.

McCandliss, B. D., Cohen, L., \& Dehaene, S. (2003). The visual word form area: Expertise for reading in the fusiform gyrus. Trends in Cognitive Sciences, 7, 293-299.

Mercure, E., Dick, F., Halit, H., Kaufman, J., \& Johnson, M. H. (2008). Differential lateralization for words and faces: Category or psychophysics? Journal of Cognitive Neuroscience, 20, 2070-2087.

OLDFIELD, R. C. (1971). The assessment and analysis of handedness: The Edinburgh Inventory. Neuropsychologia, 9, 97-113.

Picton, T. W., Bentin, S., Berg, P., Donchin, E., Hillyard, S. A., JoHnson, R., JR., ET AL. (2000). Guidelines for using human eventrelated potentials to study cognition: Recording standards and publication criteria. Psychophysiology, 37, 127-152.

Pollatsek, A., \& Well, A. D. (1995). On the use of counterbalanced designs in cognitive research: A suggestion for a better and more powerful analysis. Journal of Experimental Psychology: Learning, Memory, \& Cognition, 21, 785-794.

Price, C. J., \& Devlin, J. T. (2003). The myth of the visual word form area. NeuroImage, 19, 473-481.

Rossion, B., Joyce, C. A., Cottrell, G. W., \& Tarr, M. J. (2003). Early lateralization and orientation tuning for face, word, and object processing in the visual cortex. NeuroImage, 20, 1609-1624.

Rumsey, J. M., Horwitz, B., Donohue, B. C., Nace, K., Maisog, J. M., \& ANDREASON, P. (1997). Phonological and orthographic components of word recognition: A PET-rCBF study. Brain, 120, 739-759.

Stone, J., Leicester, L., \& Sherman, S. M. (1973). The naso-temporal division of the monkey's retina. Journal of Comparative Neurology, 150, 333-348.

Tan, L. H., Liu, H. L., Perfetti, C. A., Spinks, J. A., Fox, P. T., \& GaO, J. H. (2001). The neural system underlying Chinese logograph reading. NeuroImage, 13, 836-846.

Tan, L. H., Spinks, J. A., GaO, J. H., Liu, H. L., Perfetti, C. A., XIONG, J., ET AL. (2000). Brain activation in the processing of Chinese characters and words: A functional MRI study. Human Brain Mapping, 10, 16-27.

Tzeng, O. J. L., Hung, D. L., Cotton, B., \& Wang, S. Y. (1979). Vi- 
sual lateralization effect in reading Chinese characters. Nature, 282, 499-501.

VOYER, D. (1996). On the magnitude of laterality effects and sex differences in functional lateralities. Laterality, 1, 51-83.

Weekes, B. S., Chen, M. J., \& Lin, Y.-B. (1998). Differential effects of phonological priming on Chinese character recognition. Reading \& Writing, 10, 201-222.

Weekes, B. S., \& Zhang, B. Y. (1999). Chinese character recognition in the left and right visual fields. Brain \& Cognition, 40, 269-272.

YANG, M. J., \& CHENG, C. M. (1999). Hemisphere differences in accessing lexical knowledge of Chinese characters. Laterality, 4, 149-166.

\section{SUPPLEMENTAL MATERIALS}

A list of the stimuli used in this study may be downloaded from http:// cabn.psychonomic-journals.org/content/supplemental.

\section{NOTES}

1. In addition, Tzeng et al. (1979) showed an RVF/LH advantage in tachistoscopic recognition of Chinese two-character words.

2. Note, however, that in these characters, the mapping from a phonetic component to its pronunciation is not as fine-grained as the graphemephoneme conversion in alphabetic languages. The pronunciation of a phonetic component within a character is at the syllable level. In some cases, it has the same pronunciation as the character (i.e., regular characters); in some other cases, it shares the same pronunciation onset or rhyme with the character or has a completely different pronunciation from the character (i.e., irregular characters).

3 . We removed 2 participants since their accuracy in the digit-naming task was lower than .8. Two new participants were then recruited to replace the removed data, and they both had accuracy over 8 in the digit-naming task.

4. Note that this zero reference is based on the assumption that the head is a perfect sphere and the scalp is densely sampled, with equal space between electrodes. It has been suggested that to properly implement it, the sampling of the scalp should be reasonably dense (a minimum of 20 electrodes is required; the more electrodes, the better; see Dien, 1998; Katznelson, 1981), and the electrodes should provide a reasonable sample of sites below the $\mathrm{Fpz}-\mathrm{Oz}$ equator line (Dien, 1998;
Picton et al., 2000). Nevertheless, the implementation of this technique is usually limited by the standard EEG caps and equipment available. In the present study, a standard Neuroscan 64-channel EEG cap was used (see Hsiao et al., 2007, Figure 4, plus M1 and M2 on the left and right mastoids), and thus there were only two electrodes (M1 and M2) below the $\mathrm{Fpz}-\mathrm{Oz}$ equator line.

5. In Hsiao et al. (2007), we selected the four most prominent electrodes in each hemisphere (LH, PO7, PO5, P7, P5; RH, PO8, PO6, P8, P6) for N170 analysis. In contrast, here we selected only the two most prominent electrodes in each hemisphere (e.g., for SP characters, PO7 and $\mathrm{PO} 5$ in the $\mathrm{LH}$, paired with corresponding electrodes in the RH: PO8 and PO6), since P7 and P5 had lower N170 amplitude than did PO7 and PO5, and including P7 and P5 in the analysis made N170 amplitude of selected electrodes inhomogeneous. Indeed, if we included P7 and P5 (and paired with P6 and P8 in the RH) in the present analysis, there was a significant interaction between electrodes and the hemispheric asymmetry effect observed in SP characters $[F(3,87)=3.119, p<.05]$ : The hemispheric asymmetry effect was observed only in the two most prominent electrodes (LH, PO7 and PO5, paired with RH, PO6 and PO8), but not in the other electrodes (P7, P5, P6, and P8).

6 . Note that there were missing data for some participants since they did not have a prominent peak within the $50-\mathrm{msec}$ window around the peak latency of the grand average data in some conditions.

7. In Figure 5, there was a hemispheric asymmetry effect in the P1 component, independent of stimulus type. The functional significance of this component remains controversial. It may reflect low-level visual properties of the stimuli (Rossion, Joyce, Cottrell, \& Tarr, 2003), and its asymmetry may reflect a more RH-lateralized activation in the visual system in Chinese character processing (for more details, see Hsiao et al., 2007).

8. In Hsiao et al. (2007), the interpretation for the observed interaction between hemisphere and character type (SP vs. PS characters) in N170 amplitude was the difference in visual complexity between semantic and phonetic radicals: Phonetic radicals are usually more visually complex than semantic radicals. Nevertheless, this factor alone is not able to explain why a lateralization effect was observed in SP characters, but not in PS characters.

(Manuscript received March 22, 2010; revision accepted for publication June 24, 2010.) 